Indonesian Journal of Cardiology

Indonesian J Cardiol 202 I:42:94- 102

pISSN: 0I 26-3773 / elSSN: 2620-4762 doi: $10.30701 /$ ijc. I 144

\title{
Patent Foramen Ovale and Cryptogenic Stroke : Challenges in Diagnosis and Management
}

\author{
Dhanang Ali Yafi, Azmi.
}

Department of Cardiology and Vascular Medicine, Faculty of Medicine, Universitas PadjadjaranDr. Hasan Sadikin Hospital, Bandung

\section{Correspondence:}

Dhanang Ali Yafi. General

Practitioner. Prof. Dr. Sulianti

Saroso Infectious Disease Hospital, Jakarta, Indonesia

Email: dhanangaliyafi@gmail.com

\begin{abstract}
A patent foramen ovale is a common disorder that affects between $20-34 \%$ of the adult population. This condition is a benign finding for most people. However, In some the patent foramen ovale can open widely and enabling paradoxical embolism to transit from venous to arterial circulation, which is associated with stroke and systemic embolization. There are still unclear to date regarding the effectiveness of pharmacological anticoagulant therapy, defined as antithrombin or antiplatelet therapy, which has proven to be more beneficial for patients with patent foramen ovale and cryptogenic stroke. In addition, surgical and transcutaneous patent foramen ovale closure has been proposed for secondary prevention of stroke in patients with cryptogenic stroke with patent foramen ovale. Both catheter-based and surgical modes of closure have been shown to reduce the incidence of subsequent embolism substantially. This review will discuss the evidence regarding the relationship between patent foramen ovale and cryptogenic stroke and decision making for management strategies.
\end{abstract}

(Indonesian J Cardiol. 2021;42:94-102)

Keywords: Patent Foramen Ovale, Cryptogenic Stroke, Paradoxical Embolism, Echocardiography, Device Closure. 
Indonesian Journal of Cardiology

\section{Introdution}

7 he patent foramen ovale, a part of the disorder known as an atrial septal defect, is a remnant of normal fetal anatomy. More than half of babies will develop patent foramen ovale by six months of age. ${ }^{1}$ Although it has no clinically significant impairment, it can persist into adulthood. For most people, patent foramen ovale will remain undetected or appear only as an accidental discovery during a cardiac examination. However, some patent foramen ovales may be wide open and act as access for materials such as thrombus, air or vasoactive peptides to provide shunt from the veins to the arterial circulation - paradoxical embolism. This is associated with cryptogenic (having no other identifiable cause) stroke, systemic embolism, migraine with aura, acute limb ischemia due to embolism and decompression sickness in divers. ${ }^{2,3}$

The diameter of the patent foramen ovale (average, $4.9 \mathrm{~mm}$ ) allows passage of a sufficiently large embolism from the venous system to occlude the middle cerebral artery to reach the cerebral circulation. Worldwide annually, 345,000 patients aged 18-60 years present with patent foramen ovale and embolic stroke from undetermined sources. Young patients with cryptogenic stroke had a 2.3-fold increased relative risk of having a patent foramen ovale, compared with individuals of the same age with a clear cause of stroke, suggesting that patent foramen ovale is the causative mechanism of stroke in these patients. ${ }^{4}$

Based on transesophageal echocardiographic (TEE) screening, patients with cryptogenic stroke / transient ischemic attack (TIA) had a mean prevalence of any patent foramen ovale, patent foramen ovale associated with atrial septal aneurysm (ASA), and large patent foramen ovale of $43.2 \%, 14.5 \%$, and $19.5 \%$, respectively. The prevalence of any patent foramen ovale, patent foramen ovale with septal aneurysm, and large patent foramen ovale showed a noticable variability between younger patients $(<50$ years) versus older patients: $59.9 \%$ vs $35.2 \%, 16.3 \%$ vs $11.6 \%$, and $18.6 \%$ vs. $22.9 \%$, respectively; indicating that patent foramen ovales tend to close over time, large patent foramen ovales tend to persist into older age. ${ }^{5}$

\section{Patent Foramen Ovale - Associated Clinical Syndrome}

\section{Cryptogenic Stroke}

Patent foramen ovale is a flap-like opening between the atrial septum secundum and primum at the fossa ovalis. this opening serves as a track for blood to the systemic circulation during the womb. as pulmonary circulation increases after birth, the patent foramen ovale functionally begins to close. Complete closure of the anatomical patent foramen ovale usually occurs in about 12 months. Patent foramen ovale plays a role in increasing the risk of stroke from paradoxical embolism. The risk of cryptogenic stroke is increased in patent foramen ovale with larger defects and the presence of interatrial aneurysms, this may be due to increased in situ thrombus formation in the aneurysm tissue or because patent foramen ovales with interatrial septal aneurysms tend to have larger defects. Despite previous reports noticing paradoxical embolism via patent foramen ovale, this phenomenon as a risk factor for stroke remains difficult to prove because deep venous thrombosis was rarely detected in such patients. In one study, pelvic vein thrombus was found to be more frequent in young patients with cryptogenic stroke than in those with known the cause of stroke. These findings may serve the source of venous thrombi, particularly when the source of venous thromboembolism (VTE) was not identified. ${ }^{2}$

The relationship between patent foramen ovale and cryptogenic stroke has been introduced in the 80s of the last century. Paradoxical embolism is the suspected mechanism that occured in patent foramen ovale with stroke when a thrombus from the systemic venous circulation passes through to the systemic circulation via a right-to-left shunt. The concept of paradoxical embolism via patent foramen ovale was first conceived by Zahn in 1881, who discovered a thrombus from a uterine vein trapped in patent foramen ovale on postmortem examination. In patients with cryptogenic stroke, the rates of patent foramen ovale (59\% vs 19\%) and deep vein thrombosis ( $20 \%$ vs $4 \%$ ) were higher than patients with a stroke of known cause. ${ }^{5,6}$ In addition to paradoxical embolism, the combination of large patent foramen ovale with and atrial septal aneurysm (ASA) has been suggested as a cause of left atrial (LA) dysfunction 
predisposing to systemic thromboembolism that normalized after patent foramen ovale device closure. ${ }^{5}$

About $40 \%$ of ischemic strokes have no clear etiology and are therefore termed cryptogenic. A study conducted on 60 adult patients under 55 years with ischemic stroke compared contrast echocardiography with 100 normal subjects. The prevalence of patent foramen ovale was significantly higher in the stroke group (40\%) than in the control group (10\%) (p 0.001). The study concluded that patent foramen ovale-induced paradoxical embolism is the cause of stroke.7 The patent foramen ovale-ASA study supports these findings, in which $46 \%$ of young cryptogenic stroke patients had patent foramen ovale. 8 Cramer et al. ${ }^{6}$ evaluated young stroke patients (ages 18 to 60 years) immediately after the onset of stroke using magnetic resonance imaging (MRI) venography. Pelvic deep vein thrombosis was increased in the cryptogenic stroke population compared with controls (20\% vs $4 \%)$. The cryptogenic stroke group was younger (42 vs 49 years). The prevalence of patent foramen ovale was significantly higher in the cryptogenic stroke group than in the control group (59\% vs 19\%). A prospective study of 598 patients (ages 18 to 55 years) who had a cryptogenic stroke showed that $36 \%$ had patent foramen ovale, $1.7 \%$ had ASA, and $8.5 \%$ had both condition. Patients with patent foramen ovale and ASA who have had a stroke have a higher risk of having recurrent strokes.'

An association between prothrombotic conditions and patent foramen ovale with cryptogenic stroke has been reported. Factor V Leiden and prothrombin G20210A mutations were observed to occur more frequently in patients with patent foramen ovale and cryptogenic stroke. ${ }^{10,11}$ In one study, at least one of these two prothrombotic genotypes was significantly higher in young cryptogenic stroke patients than in agematched controls $(10.3 \%$ vs. $2.5 \%$; $p=0.008)$, with the prothrombin G20210A mutation significantly higher than factor $V$ Leiden $(8.2 \%$ vs $2.1 \%) .{ }^{12}$ The association between either this two genotypes and patent foramen ovale increased the risk of stroke by 4.7 -fold. The association between patent foramen ovale size and the presence of antiphospholipid antibodies has been reported. ${ }^{13}$ Common risk factors for venous thrombosis, such as recent surgery, traumatic injury, or use of contraceptive drugs, may increase the risk of paradoxical embolization via patent foramen ovale. ${ }^{14}$

\section{Platypnea - Orthodexia syndrome}

Platypnea-orthodeoxia syndrome consists of dyspnea and desaturation of the artery in an upright position (platypnea) with restoration in the supine position (orthodeoxia). The two components must coexist to establish this syndrome. One of the causes is an anatomical defect and the other is functional. The anatomical component must have an interatrial shunt, consist of an atrial septal defect (ASD), patent foramen ovale, fenestrated ASA, or an intrapulmonary shunt. ${ }^{15,16}$

In this syndrome there is an increase in right atrial pressure which causes a right-to-left shunt. Interestingly, blood can flow from right to left at the atrial level even when the right heart pressure is normal, as is usually the case with persistent eustachian valves. The definitive treatment for platypnea-orthodeoxia is closure of the atrial shunt. ${ }^{15,17}$

\section{Decompression Sickness}

Arterial gas embolism via ASD was first reported in scuba divers in $1986 .{ }^{18}$ Type 1 DCS consists of local joint pain, musculoskeletal pain, and / or skin rash, and type 2 DCS consists of neurological symptoms (tingling of the legs, paresthesias, severe headache with altered mental status, paraplegia, loss of consciousness, audiovestibular symptoms, and dyspnea with chest pain). Patent foramen ovale is significantly associated with type 2 DCS. ${ }^{15}$

A recent study found a strong association between patent foramen ovale sizes and DCS in 230 divers. ${ }^{19}$ Another study demonstrated the functional and anatomical characteristics of patent foramen ovale with and without DCS, this study shows that DCS is associated with right-to-left shunting at rest. Atrial septal mobility and patent foramen ovale diameter are also associated with the risk of DCS. ${ }^{20}$

\section{Migraine}

Migraine and vascular headaches are associated with patent foramen ovale. Migraine headache is a benign headache syndrome that recurs, accompanied by nausea, vomiting, and / or other symptoms of neurological dysfunction. More than 2,500,000 patients in the U.S. experiencing at least one migraine headache each week, with a lifetime prevalence of about $18 \%$. Migraine is 
a risk factor for cryptogenic stroke, notably in young patients without atherosclerotic risk factors. ${ }^{15}$

One study represented a significant association between patent foramen ovale closure and migraine improvement with aura using the Transcranial Droppler (TCD). In that study, 5 of 17 patients experience complete resolution of migraine, 10 of 17 patients admit improvement, and 2 patients had no change for 6 months after closure of the patent foramen ovale. ${ }^{21}$ Another study conducted to find the association between patent foramen ovale and migraine with or without aura. The prevalence of patent foramen ovale was $48 \%$ in patients with migraine, $23 \%$ in patients without migraine, and $20 \%$ in the control group. The differences between patients with and without migraine and patent foramen ovale were striking, as were the differences between patients with aura and the control group. However, the group without aura had no difference in patent foramen ovale prevaence compared to the control group. A recent study showed transcatheter patent foramen ovale closure led to complete resolution or a marked reduction in migraine frequency. ${ }^{22}$ In this study, has been investigated 162 patients with paradoxical brain embolism who underwent transcatheter patent foramen ovale closure. Complete migraine resolution occurred in 56\% of patients, and $14 \%$ of patients reported a significant reduction in migraine frequency. Patients reported a decrease in the mean number of migraine episodes per month by $80 \%$ after patent foramen ovale closure $(6.8 \pm$ 9.6 before closure vs $1.4 \pm 3.4$ after closure, $\mathrm{p}<0.001$ ). Another study also concluded that patent foramen ovale or ASD closure in patients with migraine headaches exhibit to migraine resolution or improvement about $76 \%$ of 89 adult patients. ${ }^{23}$

Venous blood can enter the arterial circulation directly without passing through the pulmonary circulation via the patent foramen ovale. Some chemicals and hormones such as serotonin can pass through the pulmonary circulation and directly cross the bloodbrain barrier causing migraines. ${ }^{24}$ Normally, Serotonin produced from platelet aggregation. Other evidence suggests that platelet activation and aggregation have been shown to increase in migraine patients. ${ }^{25}$ In addition, a study showed that aspirin, an anti-platelet drug can reduce platelet-fibrin complex formation and expexted to improve migraine, also has a statistically significant prophylactic effect on migraine. ${ }^{26}$
In addition, a small embolism from the systemic circulation can pass via patent foramen ovale and directly into the arterial system. This paradoxical embolism can cause tiny brain infarction, developoing low perfusion or cortical spreading depression, thereby leading to migraine attacks. ${ }^{24}$

\section{Diagnosis}

Most patients with an isolated patent foramen ovale have no symptoms. For the majority of people, the patent foramen ovale will remain undetected or accidentally discovered during a heart exam. When patent foramen ovale do accure, the patient may have a history of stroke or transient ischemic attack of undetermined etiology (cryptogenic stroke), migraine, neurologic decompression sickness (seen in patent foramen ovale experienced by a small percentage of scuba divers). ${ }^{3}$

Some imaging modalities can be used to detect patent foramen ovale, especially transthoracic echocardiography (TTE), transesophageal echocardiography (TEE), or transcranial Doppler ultrasound (TCD). ${ }^{4}$ TTE is a non-invasive examination to detect patent foramen ovale. ${ }^{27}$ TTE is the least sensitive, detecting only $50 \%$ to $60 \%$ of the patent foramen ovales found in TEE or TCD. When compared with autopsy which is the gold standard diagnosis, TEE has a sensitivity of up to $90 \%$ and a specificity above 95\%. TCD can detect 100\% of the patent foramen ovale found in TEE and detect $10 \%$ of the patent foramen ovale that is missed with TEE; TCD can even detect small patent foramen ovales that missed with TEE by perform Valsalva maneuver, and confirmed the waveforms formed during TDC. TEE and TCD are complementary. Both can detect patent foramen ovale and quantify the shunt size. TEE also able to characterizes patent foramen ovale anatomy and presence of an atrial septal aneurysm (ASA) and assesses the presence of competing proximal sources of embolism, including aortic arch atherosclerosis, atrial appendage thrombi, and signs of atrial cardiopathy. TCD uniquely quantifies the cerebral burden of paradoxical embolism, on both bubble study and during 30 minutes of monitoring for spontaneous microembolism, and assesses the presence of competing distal arterial sources of embolism. ${ }^{4}$

TTE and TEE have a considerable clinical role in patients with patent foramen ovale and cryptogenic stroke, however, the selection of these echocardiographic 
modalities should be made on a case-by-case basis. A study in ischemic stroke patients with unknown etiology (before getting an echocardiogram) evaluated patients with TTE and TEE.28 Based on the TEE results, the presence of patent foramen ovale was determined the saline bubble shunting into the left atrium in three cardiac cycles (small shunt 3-10 bubbles, moderate 1020 bubbles and large 20 bubbles), often augmented by the Valsalva maneuver that triggers the bubble to pass by increasing right atrial pressure. However due to the low sensitivity of TTE for patent foramen ovale detection, a high suspicion for patent foramen ovale with a negative TTE will often lead to ordering a TEE. Although the TEE shows a better look at cardiac structures, it is semiinvasive, with sedation that often limits or precludes the Valsalva manoeuver. ${ }^{27}$ These suggest that TEE may be superior to TTE in including or excluding a cardioembolic source for stroke; on other hand, they suggest that when a stroke etiology has not been identified using conventional means, a TEE should be considered to help identify the stroke etiology and guide stroke prevention strategies. ${ }^{28}$

TCD has been shown to be more sensitive than TEE and just as specific as TTE or TEE in emboli detection. But TCDs cannot detect additional and potentially relevant structural features such as ASA and septal mobility (features that affect shunt size characterization) nor really distinguish between intracardiac and intrapulmonary shunts such as pulmonary arteriovenous malformations. ASA is present when redundant tissue in the fossa ovale causes more than $10-15 \mathrm{~mm}$ of bulging into the left or right atrium during respiration, and may indicate a greater recurrent stroke risk compared with patent foramen ovale alone. Tobe et al found that a shunt grade determined by TCD can be a stronger predictor of TIA or stroke than shunt detection by TEE, and that TEE missed $15 \%$ of the shunts caught by TCD, and of those $40 \%$ were large shunts (grade 3 and higher). But, TCD should not replace echocardiographic modalities in patent foramen ovale detection and other shunt features, but can be a complementary and highly sensitive modality when performed by a properly trained and experienced operator. ${ }^{27}$

Despite the strong evidence shows there is an association between patent foramen ovale and the risk of stroke, confirming a causal relationship in a given patient is still challenging. Even in those with cryptogenic
Table 1. The Risk of Paradoxical Embolism (RoPE) score. ${ }^{29}$

\begin{tabular}{lc}
\hline \multicolumn{1}{c}{ Name } & Value \\
\hline Vascular risk factors & \\
No Hypertension & 1 \\
No Diabetes Mellitus & 1 \\
No prior stroke or transient ischaemic attack & 1 \\
Non-smoker & 1 \\
Age & \\
$18-29$ & 5 \\
$30-39$ & 4 \\
$40-49$ & 3 \\
$50-59$ & 2 \\
$60-69$ & 1 \\
$\geq 70$ & 0 \\
Stroke features & \\
Cortical infarction & 1 \\
\hline
\end{tabular}

stroke, at least one third of patent foramen ovales discovered are likely to be incidental and closure of an incidental patent foramen ovale would expose patients to procedural and device-related risks while leaving the actual cause of stroke unrevealed. Estimating the probability of a patent foramen ovale being embolism related is based on patient's clinical profile (younger age, absence of atherosclerosis risk factors, a higher RoPE scope, pulmonary hypertension/ obstructive sleep apnea), cerebral infarct pattern (typical of embolism), anatomical patent foramen ovale features (large shunt, atrial septal aneurysm), and conditions potentially predisposing to/precipitating paradoxical embolism through patent foramen ovale. ${ }^{5}$

\section{Clinical Profile}

Worldwide, about 345,000 patients aged 18-60 years with a patent foramen ovale and an embolic stroke of otherwise undetermined source preset each year. Younger patients with a cryptogenic stroke have a 2.3fold increased relative risk of having a patent foramen ovale, compared with age-matched individuals with a stroke of a clear cause, suggesting 73\% likelihood that patent foramen ovale is the mechanism of the stroke in those patients. $^{5}$

Several studies have examined clinical hints that can be used to determine how likely patent foramen ovale is causing paradoxical embolism. History of diseases such as pulmonary embolism or DVT, migraine, stroke symptoms preceded by the Valsalva maneuver, and 
symptoms of stroke / TIA coincide on waking from sleep preceded by sleep apnea has been associated as a major risk factor for cerebrovascular events associated with patent foramen ovale. ${ }^{27}$ The development of atheroscelrotic plaque was linearly correlated with the incidence of ischemic stroke. However, patent foramen ovale as a cause of stroke is supported by the absence of risk factors for plaque development such as younger age, absence of hypertension, hyperlipidemia, diabetes mellitus, and smoking. ${ }^{4}$

The difficulty in attributing patent foramen ovale for the culprit cause of stroke has led to the development of the Risk of Paradoxical Embolism (RoPE) score for cryptogenic stroke, which was drawn from a database of 3023 patients with cryptogenic stroke, who had previously been examined using TEE or TCD, the cryptogenic stroke defined by the Trial. Org 10172 in Acute Stroke (TOAST). ${ }^{27,29}$ The RoPE score is calculated from the following variables: younger age, the presence of a cortical stroke on neuro imaging, absence of diabetes, absence of hypertension, non smoker, and no prior stroke or TIA. The highest RoPE score, that indicating a higher likely-hood patent foramen ovale related stroke rather than incidental, can be found in case of the youngest patient with superficial strokes and without atherosclerosis risk factors. RoPE score is helpful and practical, but should always be used in conjunction with other parameters because it is only modestly validated and does not account for high-risk morphological features of the patent foramen ovale. ${ }^{30}$ There was few external validation studies, one of small study identified a RoPE score $>7$ as a cutoff point that indicated patent foramen ovale is more likely to be stroke related. ${ }^{31}$ In other cohort study of a cryptogenic stroke, the prevalence of patent foramen ovale-related right-to-left shunt was $50-56 \%$ in patients with RoPE scores $<7$ compare with $79 \%$ in those with a score $\geq 7$. Additionally, a higher RoPE score correlated modestly with the severity of right-to-left shunt..$^{32}$ Another studies use a score $>5$ or $>6$ to classify patent foramen ovale as stroke related. ${ }^{33,34}$

\section{Cerebral Infact Pattern}

Stroke was only called as cryptogenic after history, exam, routine labs and extensive testing including; initial neurovascular assessment (CT/MRI, vascular Imaging), initial cardiac assessment does not identified the stroke mechanism. ${ }^{28}$ Association between patent foramen ovale and cryptogenic stroke has been established in the 80 s of the last century. Paradoxical embolism is the suggested mechanism patent foramen ovale related to stroke and occurs when a thrombus from the systemic venous circulation passes to the systemic arterial circulation trhough a right-to-left shunt. ${ }^{5}$ The mean diameter of persisting patent foramen ovales is $4.9 \mathrm{~mm}$ (range about $1-19 \mathrm{~mm}$ ), more than sufficient to allow passage of emboli large enough to occlude the middle cerebral artery stem $(3 \mathrm{~mm})$ and major cerebral cortical branches $(1 \mathrm{~mm}){ }^{4}$

Noncontrast head CT highly effective for excluding intracranial hemorrhage; however, it is poor at best for identifying small infarcts. MRI is better than CT in detecting ischemic stroke. when available, MRI should be preferred over CT for the initial imaging of the stroke patient. Diffusion-weighted found in MRI may also help identify a stroke mechanism; for example, multiple lesions in different vascular territories may suggest a cardioembolic origin. Otherwise, scattered lesions to a single vascular distribution suggest largeartery atherosclerosis. Other study have suggested that cryptogenic stroke patients who have clinical and CT evidence of one ischemic lesion should take a subsequent MRI assessment to further delineate potential causes. ${ }^{35,36}$ The probability that stroke is related to patent foramen ovale is increase in case of a cortical infarct (suggesting an embolic mechanism). ${ }^{5}$

\section{Management of Patent Foramen Ovale-Related Cryptogenic Stroke}

In general there are two treatment options for secondary prevention of recurrent stroke in cryptogenic ischemic stroke patients with patent foramen ovale include medical treatment (alone or in combination, antiplatelet and anticoagulant therapy) and patent foramen ovale closure (surgical patent foramen ovale closure, and percutaneous patent foramen ovale closure). ${ }^{4}$

\section{Medical Treatment}

Acording to pathophysiological standpoint, anticoagulation might be better than antiplatelet therapy in purpose to preventing patent foramen ovale-related stroke, as anticoagulants is superior prevent thrombi 
arising in veins. Nevertheless, anticoagulation is also associated with increased bleeding event, and comparative studies have shown only modest evidence of an efficacy advantage. ${ }^{5}$ In addition, bleeding complications were significantly more frequent among anticoagulated patients. Compared with older anticoagulants, nonvitamin $\mathrm{K}$-dependent oral anticoagulants are promising options, more reduced bleeding rates, and comparable efficacy in prevention of DVT, but in patients with patent foramen ovale have not been studied yet. ${ }^{4}$ While antiplatelet therapy applied in clinical trials is variable, including of acetylsalicylic acid, clopidogrel, and/or extended-release dipyridamole. Currently available evidence suggests that anticoagulants may be superior to antiplatelet therapy. ${ }^{5}$

Among the medical therapy options, US national practice guidelines weakly endorsed antiplatelet therapy as preferred. Although physiological reasoning suggests that anticoagulation might be superior to antiplatelet therapy, as anticoagulants better avert stasis thrombi arising in veins, anticoagulation is also associated with increased bleeding, and comparative studies are only weakly suggestive of an efficacy advantage. There was two randomized trials comparing anticoagulant against antiplatelet therapy in subgroups of patients with patent foramen ovales and cryptogenic ischemic stroke found non-significant efficacy differences: PICSS trial (patent foramen ovale in Cryptogenic Stroke Study; hazard ratio $[\mathrm{HR}], 0.52 ; 95 \%$ confidence interval [CI], 0.16-1.67; $\mathrm{P}=0.28)^{37}$ and CLOSE trial (Patent Foramen Ovale Closure or Anticoagulants versus Antiplatelet Therapy to Prevent Stroke Recurrence; HR, 0.44; 95\% CI, 0.111.48; $\mathrm{P}=0.18) .{ }^{38}$

Patent foramen ovale and deep vein thrombosis (DVT) are both common findings at the same time. Currently AHA/ASA Stroke Prevention in Patients with Stroke or TIA Guidelines recommend the following ; There are insufficient data to establish whether anticoagulation is equivalent or superior to aspirin for secondary stroke prevention in patients with patent foramen ovale (Class IIb; Level of Evidence B). For patients with an ischemic stroke or TIA and a patent foramen ovale who are not undergoing anticoagulation therapy, antiplatelet therapy is recommended. (Class I, Level of Evidence B) For patients with an ischemic stroke or TIA and both a patent foramen ovale and a venous source of embolism, anticoagulation is indicated, depending on stroke characteristics (Class I; Level of Evidence A). When anticoagulation is contraindicated, an inferior vena cava filter is reasonable (Class IIa; Level of Evidence C). ${ }^{28}$

\section{Patent Foramen Ovale Closure}

Surgical closure of the patent foramen ovale requiring thoracotomy and cardiopulmonary bypass is rarely performed as independent therapy. It may be an option if the patient is undergoing heart surgery for other indications. In observational series, open surgical closure has a low rate of perioperative mortality, but can leave sequelae of morbidity including AF, pericardial effusion, postoperative bleeding, infection, and postpericardiotomy syndrome. The annual incidence rate for recurrent stroke or transient ischemic attack ranges from $0 \%$ to $9 \%$. Recurrence of cerebral ischemia associated with incomplete closure of the patent foramen ovale with an open surgical approach occurs in up to $73 \%$ of patients. ${ }^{4}$

The percutaneous patent foramen ovale closure device is a minimally invasive approach to anatomical management that has advanced in the last quarter century since the first trials were carried out on humans. Device designs differ in important ways that affectease of delivery, efficacy in achieving complete closure, and adverse effects. ${ }^{4} \mathrm{~A}$ large number of devices with varying shapes and sizes have been marketed, the need for evidence from randomised controlled trials prior to approval means fewer devices have been approved by the Food and Drug Administration. Most devices are of double-disc design, connected by a short waist. The Gore Septal Occluder (WL Gore \& Associates) and the AMPLATZER patent foramen ovale Occluder (Abbott Vascular) are two of the more commonly used devices (Picture 1). The Gore Septal Occluder is constructed from five nitinol wires covered with expanded polytetrafluoroethylene, early clinical experience has shown that it is a versatile device with easy deployment, high procedural success rates and low complication rates. The AMPLATZER patent foramen ovale Occluder is also a nitinol-based device. These devices are used most often in randomized controlled trials, and the evidence for their effect is very reliable. The operator must gain experience using different devices to find out the best outcome for the patient. ${ }^{3}$ 
Recent investigations have shown that closure of patent foramen ovale, particularly with atrial septal aneurysms and / or large interatrial shunts, can reduce the risk of recurrent stroke compared with pharmacological treatments. However, it remains challenging to risk stratify patients with suspected patent foramen ovaleassociated stroke and to decide whether device closure is indicated or not..$^{5}$ Patients with cryptogenic stroke or TIA and patent foramen ovale without evidence of DVT, available data do not support the benefit for patent foramen ovale closure (Class III; Level of Evidence A). In patent foramen ovale and DVT settings, patent foramen ovale closure by a transcatheter device might be considered, depending on the risk of recurrent DVT (Class IIb; Level of Evidence C). ${ }^{28}$

The antithrombotic regimen after device closure is still uncertain. ${ }^{3}$ Hence, routine dual antiplatelet therapy (Aspirin and clopidogrel) is recommended for 1-6 months after patent foramen ovale closure, and single antiplatelet therapy (usually clopidogrel $75 \mathrm{mg}$ daily) afterwards for at least 5 years., ${ }^{3,5}$ Transthoracic echocardiography (TTE) should be performed prior to discharge and 6 weeks after device insersion to eliminate pericardial effusion and device embolisation. Potential successful rate of closure are high using modern devices, and the main objective is sealing the patent foramen ovale flap valve opening. Total closure depends on device endothelialization and can take up to 6 months, after which a repeat bubble study should be performed to confirm complete closure has occurred. ${ }^{3}$

\section{Conclusion}

In this article, patent foramen ovale management focused on cryptogenic stroke has been discussed, along with scientific evidence of either pharmacological or intervention for patent foramen ovale closure. Clinical knowledge and attention to detail in indication for the procedures and risks to patients are required with the help from experienced interventional cardiologists.

\section{References}

1. Connuck D, Sun JP, Super DM, Kirchner HL, Fradley LG, Harcar-Sevcik RA, Salvator A, Singer L, Mehta SK. Incidence of patent ductus arteriosus and patent foramen ovale in normal infants. Am J Cardiol. 2002;89:244-247.
2. Hampton T, Alsaleem M, Murphy-Lavoie HM. Patent Foramen Ovale. [Updated 2021 Mar 3]. In: StatPearls [Internet]. Treasure Island (FL): StatPearls Publishing; 2021 Jan-. Available from: https://www.ncbi.nlm.nih. gov/books/NBK493151/?report=classic.

3. Giblett JP, Abdul-Samad O, Shapiro LM, Rana BS, Calvert PA. patent foramen ovale closure in 2019. Interv Cardiol Rev. 2019;14:34-41.

4. Saver JL, Mattle HP, Thaler D. patent foramen ovale closure versus medical therapy for cryptogenic ischemic stroke a topical review. Stroke. 2018;49:1541-1548.

5. Abdelghani M, El-Shedoudy SAO, Nassif M, Bouma BJ, De Winter RJ. Management of Patients with patent foramen ovale and Cryptogenic Stroke: An Update. Cardiol. 2019;143:62-72.

6. Cramer SC, Rordorf G, Maki JH, Kramer LA, Grotta JC, Burgin WS, Hinchey JA, Benesch C, Furie KL, Lutsep HL, Kelly E, Longstreth WTJ. Increased pelvic vein thrombi in cryptogenic stroke: results of the Paradoxical Emboli from Large Veins in Ischemic Stroke (PELVIS) study. Stroke. 2004;35:46-50.

7. Lechat P, Mas JL, Lascault G, Loron P, Theard M, Klimczac M, Drobinski G, Thomas D, Grosgogeat Y. Prevalence of patent foramen ovale in patients with stroke. N Engl J Med. 1988;318:1148-1152.

8. Lamy C, Giannesini C, Zuber M, Arquizan C, Meder JF, Trystram D, Coste J, Mas JL. Clinical and imaging findings in cryptogenic stroke patients with and without patent foramen ovale: the patent foramen ovale-ASA Study. Atrial Septal Aneurysm. Stroke. 2002;33:706-711.

9. Mas JL, Arquizan C, Lamy C, Zuber M, Cabanes L, Derumeaux G, Coste J. Recurrent cerebrovascular events associated with patent foramen ovale, atrial septal aneurysm, or both. N Engl J Med. 2001;345:17401746.

10. Karttunen V, Hiltunen L, Rasi V, Vahtera E, Hillbom M. Factor V Leiden and prothrombin gene mutation may predispose to paradoxical embolism in subjects with patent foramen ovale. Blood Coagul fibrinolysis an Int J Haemost Thromb. 2003;14:261-268.

11. Lichy C, Reuner KH, Buggle F, Litfin F, Rickmann $\mathrm{H}$, Kunze A, Brandt T, Grau A. Prothrombin G20210A mutation, but not factor V Leiden, is a risk factor in patients with persistent foramen ovale and otherwise unexplained cerebral ischemia. Cerebrovasc Dis. 2003;16:83-87.

12. Botto N, Spadoni I, Giusti S, Ait-Ali L, Sicari R, Andreassi MG. Prothrombotic mutations as risk factors for cryptogenic ischemic cerebrovascular events in young subjects with patent foramen ovale. Stroke. 2007;38:2070-2073.

13. Belvís R, Santamaría A, Martí-Fàbregas J, Leta RG, Cocho D, Borrell M, Fontcuberta J, Martí-Vilalta JL. 
patent foramen ovale and prothrombotic markers in young stroke patients. Blood Coagul fibrinolysis an Int J Haemost Thromb. 2007; 18:537-542.

14. Di Tullio MR, Homma S. patent foramen ovale and stroke: What should be done? Curr Opin Hematol. 2009;16:391-396.

15. Hara H, Virmani R, Ladich E, Mackey-Bojack S, Titus J, Reisman M, Gray W, Nakamura M, Mooney M, Poulose A, Schwartz RS. patent foramen ovale: current pathology, pathophysiology, and clinical status. J Am Coll Cardiol. 2005;46:1768-1776.

16. Cheng TO. Platypnea-orthodeoxia syndrome: etiology, differential diagnosis, and management. Catheter Cardiovasc Interv Off J Soc Card Angiogr Interv. 1999;47:64-66.

17. Cheng TO. Transcatheter closure of patent foramen ovale: a definitive treatment for platypnea-orthodeoxia. Catheter Cardiovasc Interv Off J Soc Card Angiogr Interv. 2000;51:120.

18. Wilmshurst PT, Ellis BG, Jenkins BS. Paradoxical gas embolism in a scuba diver with an atrial septal defect. Br Med J (Clin Res Ed). 1986;293:1277.

19. Torti SR, Billinger M, Schwerzmann M, Vogel R, Zbinden R, Windecker S, Seiler C. Risk of decompression illness among 230 divers in relation to the presence and size of patent foramen ovale. Eur Heart J. 2004;25:1014-1020.

20. Cartoni D, De Castro S, Valente G, Costanzo C, Pelliccia A, Beni S, Di Angelantonio E, Papetti F, Vitali Serdoz L, Fedele F. Identification of professional scuba divers with patent foramen ovale at risk for decompression illness. Am J Cardiol. 2004;94:270273.

21. Morandi E, Anzola GP, Angeli S, Melzi G, Onorato E. Transcatheter closure of patent foramen ovale: a new migraine treatment? J Interv Cardiol. 2003;16:39-42.

22. Reisman M, Christofferson RD, Jesurum J, Olsen J V, Spencer MP, Krabill KA, Diehl L, Aurora S, Gray WA. Migraine headache relief after transcatheter closure of patent foramen ovale. J Am Coll Cardiol. 2005;45:493-495.

23. Azarbal B, Tobis J, Suh W, Chan V, Dao C, Gaster R. Association of interatrial shunts and migraine headaches: Impact of transcatheter closure. J Am Coll Cardiol. 2005;45:489-492.

24. Wilmshurst P, Nightingale S. Relationship between migraine and cardiac and pulmonary right-to-left shunts. Clin Sci (Lond). 2001;100:215-220.

25. Borgdorff P, Tangelder GJ. Migraine: Possible Role of Shear-Induced Platelet Aggregation With Serotonin Release. Headache J Head Face Pain. 2012;52:12981318.

26. Grotemeyer K-H, Scharafinski H-W, Schlake H-P, Husstedt IW. Acetylsalicylic Acid vs. Metoprolol in Migraine Prophylaxis - A Double-Blind Cross-Over
Study. Headache J Head Face Pain. 1990;30:639-641.

27. Yuan K, Kasner SE. patent foramen ovale and cryptogenic stroke: Diagnosis and updates in secondary stroke prevention. Stroke Vasc Neurol. 2018;3:84-91.

28. Understanding Diagnosis and Treatment of Cryptogenic Stroke: A Health Care Professional Guide. Am Stroke Assoc. 2015:24.

29. Kent DM, Ruthazer R, Weimar C, Mas J-L, Serena J, Homma S, Di Angelantonio E, Di Tullio MR, Lutz JS, Elkind MS V, Griffith J, Jaigobin C, Mattle HP, Michel P, Mono M-L, Nedeltchev K, Papetti F, Thaler DE. An index to identify stroke-related vs incidental patent foramen ovale in cryptogenic stroke. Neurology. 2013;81:619-625.

30. Pristipino C, Sievert H, D’Ascenzo F, Louis Mas J, Meier B, Scacciatella P, et al. European position paper on the management of patients with patent foramen ovale. General approach and left circulation thromboembolism. Eur Heart J. 2019;40:3182-3195.

31. Prefasi D, Martínez-Sánchez P, Fuentes B, DíezTejedor E. The utility of the RoPE score in cryptogenic stroke patients $\leq 50$ years in predicting a stroke-related patent foramen ovale. Int J stroke Off J Int Stroke Soc. 2016;11:NP7-8.

32. Wessler BS, Kent DM, Thaler DE, Ruthazer R, Lutz JS, Serena J. The RoPE Score and Right-to-Left Shunt Severity by Transcranial Doppler in the CODICIA Study. Cerebrovasc Dis. 2015;40:52-58.

33. Braemswig TB, Usnich T, Scheitz JF, Erdur H, Fiebach JB, Audebert HJ, Endres M, Nolte CH. Early Recurrent Ischemic Lesions in Patients With Cryptogenic Stroke and patent foramen ovale: An Observational Study. Front Neurol. 2018;9:996.

34. Thaler DE, Ruthazer R, Weimar C, Mas J-L, Serena J, Di Angelantonio E, Papetti F, Homma S, Mattle HP, Nedeltchev K, Mono M-L, Jaigobin C, Michel P, Elkind MS V, Di Tullio MR, Lutz JS, Griffith J, Kent DM. Recurrent stroke predictors differ in medically treated patients with pathogenic vs. other patent foramen ovales. Neurology. 2014;83:221-226.

35. Yaghi S, Elkind MS V. Cryptogenic stroke: A diagnostic challenge. Neurol Clin Pract. 2014;4:386-393.

36. Kang D-W, Chalela JA, Ezzeddine MA, Warach S. Association of ischemic lesion patterns on early diffusion-weighted imaging with TOAST stroke subtypes. Arch Neurol. 2003;60:1730-1734.

37. Homma S, Sacco RL, Di Tullio MR, Sciacca RR, Mohr JP. Effect of medical treatment in stroke patients with patent foramen ovale: patent foramen ovale in Cryptogenic Stroke Study. Circulation. 2002; 105:2625-2631.

38. Mas J-L, Derumeaux G, Guillon B, Massardier E, Hosseini $\mathrm{H}$, Mechtouff $\mathrm{L}$, et al. patent foramen ovale Closure or Anticoagulation vs. Antiplatelets after Stroke. N Engl J Med. 2017;377:1011-1021 